\title{
Nurses' Use of Non-pharmacological Interventions in Managing Common Risk Factors of Delirium in Critically Ill Patients
}

\author{
Nahla Shaaban Khalil \\ Professor at Critical Care and Emergency Nursing, Cairo University
}

Corresponding author: Nahla Shaaban Khalil, Professor at Critical Care and Emergency Nursing, Cairo University.

Received date: March 12, 2021 ; Accepted date: March 25, 2021 ; Published date: April 05,2021

Citation: Nahla S Khalil (2021) Nurses' Use of Non-pharmacological Interventions in Managing Common Risk Factors of Delirium in Critically Ill Patients. J Clinical Research and Reports, 7(3); DOI:10.31579/2690-1919/151

Copyright: () 2021 Nahla S Khalil, This is an open access article distributed under the Creative Commons Attribution License, which permits unrestricted use, distribution, and reproduction in any medium, provided the original work is properly cited.

\begin{abstract}
Incidence of delirium represented $32.3 \%$ since long in ICU settings, it might be higher. Other research showed the prevalence of delirium as high as $77 \%$ in ventilated burn patients. Incidence of delirium represented $32.3 \%$ since long in ICU settings, it might be higher. Other research showed the prevalence of delirium as high as $77 \%$ in ventilated burn patients. The incidence of delirium in the ICU ranged from $45 \%$ to $87 \%$, this ratio appeared be different to the studied population exclusively to mechanically ventilated patients.
\end{abstract}

Key words: delirium; ventilated patients; impaired vision; sleep; hallucinations; patient characteristics; detective tools; benzodiazepine

\section{Introduction}

Incidence of delirium represented $32.3 \%$ since long in ICU settings, it might be higher. Other research showed the prevalence of delirium as high as $77 \%$ in ventilated burn patients. The incidence of delirium in the ICU ranged from $45 \%$ to $87 \%$, this ratio appeared be different to the studied population exclusively to mechanically ventilated patients. On the other hand, other studies found that the incidence of delirium in mechanically ventilated patients' was $83 \%$ [29].

Delirium manifested as a collection of symptoms that appeared suddenly or gradually of the patients. These symptoms have been organized into two categories: cognitive and behavioral groups. Cognitive symptoms included disturbances level of consciousness, attention, and loss of near memories, impaired vision and perseveration. But the behavioral symptoms included disturbance of sleep, irritability, hallucinations, and delusions. The manifestations of delirium can be different among patients. Some patients may manifest somnolence and sudden coma, others appeared to be anxious, disruptive, and combative [16].

There are several risk factors for delirium which grouped into predisposing baseline, and hospital based factors. The baseline factors presented before patient admission to ICU and were difficult to monitor, but the hospital based factors happened through the duration of critical illness. It involved factors through the acute illness and modifiable by preventive or therapeutic intervention [16]. Predisposing factors are patient characteristics present before ICU admission that affect a patient's vulnerability for developing delirium during hospitalization such as: old age over 65 years especially male gender, cognitive impairment, previous history of delirium, immobility, cases of dehydration, poor nutrition, addict, treatment with anticholinergic and psychoactive drugs. In addition, patients with hearing/visual impairment and functional impairment are at high risk for developing delirium [2].

Precipitating Factors (Hospital- Based) included acute illness or systemic disease as infections, hypoxia, shock, medication effect of sedative(benzodiazepines and opiates), hypnotics, narcotics, anticholinergic drugs, alcohol or drug withdrawal, admission to ICU, presence of invasive procedures (urinary, vascular catheters and gastric tubes), use of physical restraints, environmental conditions (lack of daylight, isolation and lack of visitors), pain, emotional stress, and prolonged sleep deprivation $[3,17,25]$.

Delirium associated with patients undergoing coronary artery bypass graft surgery and systemic corticosteroids with acute lung injury because of increasing level of plasma cortisol before surgery. Also, bleeding, hematocrit more than $30 \%$, and transfusion of blood during the operation period have been suggested to be risk factors for postoperative delirium. The medical staff should consider these factors before surgery to prevent delirium $[22,34,18,11]$.

Benzodiazepines and Dexmedetomidine (sedative medications) were considered as independent risk factors for delirium in ICU patients. Based on these findings, the (American Psychiatric Association, 2013) guidelines considered that benzodiazepines as a risk factor for the development of delirium. Also, antihistamines and furosemide caused delirium among medications which used in the ICU because of its effect on central anticholinergic $[14,2,9]$.

In Intensive Care Unit (ICU) assessing delirium patients can be difficult because of endotracheal intubation, using of sedatives, 
endotracheal tube and complexity of delirium diagnosis tools. Without a screening tool, most cases are missed by ICU physicians and ICU nurses. Because of these barriers, some detective tools have been improved for diagnosis of delirium and medical staff trained well on its [28].

The American College of Critical Care Medicine (ACCM) recommended that most patients in ICU should be tested for delirium at least once per each shift as less frequent screening or screening only on patient admission missed prognosis onset of delirium and evaluated for sedation level every (4-6) hours [7]. There are two tools used to assess sedation level such as Richmond Agitation Sedation Scale (RASS), and Riker Sedation Agitation Scale (SAS).

Both ICDSC and CAM-ICU have been validated as reliable tools for the diagnosis of delirium. As they are based upon the Diagnostic and Statistical Manual of Mental Disorders (DSM-IV) criteria for delirium, so it assisted medical staff in ICU to detect delirium early and rapidly and it is modified for nonverbal and mechanically ventilated patients [7]. A difference between the scales is that the CAM-ICU assessment focused on specific point in time whereas the ICDSC assessment included information from the previous 24 hours [23]. The CAM-ICU, adapted from the Confusion Assessment Method, was designed for use in intubated patients and mechanically ventilated ICU patients. It's sensitivity from $93 \%$ to $100 \%$ and specificity from $89 \%$ to $100 \%$ for delirium [15].

The first step in the Non-Pharmacological management of delirium is to recognize its presence by using of an effective assessment tool, and then review the delirium risk factors in causes that may be correctable. Because delirium is a multi-factorial syndrome, it should be treated with a multi-component protocol, which focused on the following: cognitive stimulation, sleep promotion, early mobilization, correct dehydration and vision/hearing impairment. It has been shown that multifactorial interventions are effective and decreases the duration of delirium [1].

Cognitive stimulation: The role of Critical Care Nurse (CCN) is to perform re-orientation of patients to time, person, and place. Provision of photographs, pictures, calendars, television, newspapers, and radio will help patients to relax and provide orientation or assist in reorientation to new environments. Patient recognition to time, date, and hospital name must be applied. The CCN should encouraged psychological support from patient's family, putting favorite photos or things at bedside, and explain any procedures for the patient [8].

Sleep promotion: Critical alarms in ICU such as: noise, strong light, medications, alarms of mechanical ventilator and daily patient care at inappropriate times of the day have been associated with sleep disturbances. It is believed that sleep disturbance in intensive care units is a cause of delirium so, sleep-promoting interventions should be used as part of routine care $[12,33,19]$. So, the nurse should use some measures for promoting sleep such as: minimizing night time sleep disruptions, elimination of unnecessary noise, natural light exposure at daytime, minimization of artificial light exposure at night, temperature control of environment not too cold or too hot, and encourage the patients to use ear plugs during night to promote sleep $[24,7,20]$.

Early mobilization: Immobilization is a risk factor for delirium in ICU. Most mechanically ventilated patients can't be mobilized, but changing position and motion should be encouraged by the Critical Care Nurse (CCN) as possible. Physical restraints shouldn't be used and other causes of immobility should be identified as possible. All catheters and
Lines were considered to be a barrier to mobile patients along with more simple practical factors such as, the need for extra staff to move patients and specialist equipment to get them out of bed according to early mobility protocol as previous $[25,33]$.

Vision/hearing impairment: The $\mathrm{CCN}$ should encourage patients for using eyeglasses and magnifying lenses, having patients use hearing aids and removing earwax, by using simple instructions and maintain frequent eye to eye contact with patients. Sensory impairments, such as vision and hearing loss, should be minimized by using spectacles or hearing aids. Physical restraints should be avoided as it lead to decreased patient's mobility, increased agitation, increased risk of injury, and prolonged time of delirium. On the other hand, an environment with minimal noise allowed patients long period of sleep at night and it was important in the management of delirium [16].

Mental status may be deteriorated and become worse with mild degree of dehydration ended with delirium and causes memory loss. Nurses should monitor the symptoms of severe dehydration that appeared in the form of dry mouth and lips, sunken eyes, altered mental status and diminished urine output. This is a threatening medical emergency that induce delirium and if not treated well may lead to death. Inability to recognize signs of dehydration exposes the critically ill patients to become increasingly and chronically dehydrated, which is a risk factor to delirium [32]. As a result, risk of delirium will be reduced by giving patient adequate fluid intake. Water reduces critically ill patients' risk for becoming delirious. Also milk, vegetable or fruit juice, and soup are considered to be healthy fluid choices. Carbonated and caffeinated drinks should be withheld due to their diuretic effect. The body requires water to purify alcoholic beverages from the body. So that, more water consumption is required to balance the dehydrating effects of unhealthy drinks. Taking healthy drinks is as essential as eating healthy foods [10].

The critical care nurses (CCNs) are the most important key in the ICU who improve patients' quality of care and outcomes through detecting delirium early, determining the causes of delirium, and promoting knowledge of care. Critically ill patients are at a great risk for the development of delirium in the ICU. Complications with delirium can be avoided by accurate identification and prompt modification of the risk factors of delirium and appropriate interventions will decrease the severity and time of delirium [5].

Critical Care Nurses have an essential and principal role in reducing of delirium in the ICU patients such as: toleration from sedation at daily round, improving the communication and mobility skills, optimizing pain management by performing cognitive stimulation several times each day, restoring the sleep-wake cycle, performing early mobilization, visual and auditory stimulation, encouraging patients for using eye glasses and magnifying lenses, and hearing aids, removing earwax and correcting dehydration rapidly when happing [16].

Also, ICU settings must have a strategy for the assessment of delirium. There are many validated tools that utilized to monitor the potential risk factors and detect the delirium early such as: (CAM-ICU or ICDSC) [6].These strategies including the following: decreasing benzodiazepine usage, titration sedation, daily awakening trials, performing early progressive mobility and exercise for all critically ill patients, evaluating patients for causes of delirium including medications notably benzodiazepines) [26]. 
The health care providers must have a goal from sedation and agitation scale that maintain patient calm and cooperative. Every day at morning during staff rounds, the $\mathrm{CCN}$ will report the medical team of the patient's:" target" sedation level, actual sedation and agitation score, delirium score, sedative and analgesic medication that the patient administered. There are some types of valid and reliable tools that can be used to facilitate goal-directed titration from sedationas: the Richmond Agitation Sedation Scale (RASS), and Sedation Agitation Scale (SAS) [27].

Delirium will be managed well when the medical team identified and treated the cause of the disease each daily rounds, and discussed the possible causes of the patient's delirium. It's a useful key that collected all possible causes of delirium in ICU [21] such as: toxic situations and medications, congestive heart failure, shock, dehydration, new organ failure (e.g., liver, kidney), deliriogenic medications as: (benzodiazepines, anticholinergic medications, and steroids), Hypoxemia, Infection and sepsis (nosocomial), inflammation, immobilization. Non-pharmacological interventions, and potassium or other electrolyte imbalance $[27,31]$

The medical care team assessed the patient's ability for mobilization. This team included a physical therapist who assessed the patient's physical ability to move, $\mathrm{CCN}$ who assessed physiologic status, and a respiratory therapist who is responsible for maintaining the patient's airway. In addition, the physician tested the patient for any complications that disturbed patients' ability to move [26].

The ACCM guidelines 2013 advocated the importance of early mobilization to reduce the incidence and duration of delirium. More researches involved non ICU patients recommended that early mobilization increased synaptic transmission and neurotransmitter release, promoted neurogenesis, and improving cognitive function as part of its neuro protective effect 13,4$]$.

Also, Physical restraints should be minimized during mechanical ventilation. However, it has been reported to increase delirium and agitation and the risk of unplanned extubation. So; the CCN should use physical restraints in appropriate situations for the shortest possible duration not for a routine. If restraints are needed, the $\mathrm{CCN}$ should use it in combination with sedation medication because the physical restraint can cause agitation $[24,21,14]$.

Non-pharmacological interventions for managing delirium in critically ill patients enable the nurses better to detect and manage delirium early. Non pharmacological Interventions could also be individualized to identify and get rid of risk factors specific to that patient, e.g. specific measures to control pain or promote the patient' hydration status . Various non-pharmacological interventions may act together in attempt to release the risk factors simultaneously through the use of evidenced protocols and nurse education. Managing delirium effectively may relieve physical and psychological the distress that delirium causes, minimizing the accompanied complications such as falls or reducing the severity of delirium among patients.

\section{References}

1. Banh HL. Management of delirium in adult critically ill patients: an overview. J Pharm Pharm Sci. 2012; 15(4):499509. doi: 10.18433/j3pk69. PMID: 23106953.

2. Barr J, Fraser G L, Puntillo K, Ely E W, Gélinas C, Dasta J F, ... \& Jaeschke R. (2013). Clinical practice guidelines for the management of pain, agitation, and delirium in adult patients in the intensive care unit, Critical Care Med. 41: (1), 263- 306.

3. Brummel NE, Girard TD (2013). Preventing delirium in the intensive care unit. Crit Care Clin. 2013 Jan; 29(1):51-65. doi: 10.1016/j.ccc.2012.10.007. PMID: 23182527; PMCID: PMC3508697.

4. Bryczkowski SB, Lopreiato MC, Yonclas PP, Sacca JJ, Mosenthal AC (2014). Delirium prevention program in the surgical intensive care unit improved the outcomes of older adults. J Surg Res. 2014 Jul; 190(1):280-288. doi: 10.1016/j.jss.2014.02.044. Epub 2014 Feb 28. PMID: 24666988.

Cole, M. G. et al. (2013c). Symptoms of delirium predict incident delirium in older long-term care residents. International Psychogeriatrics, 25, 887-894.

5. Colombo R, Corona A, Praga F, Minari C, Giannotti C, Castelli A, Raimondi $F$ (2012). A reorientation strategy for reducing delirium in the critically ill. Results of an interventional study. Minerva Anestesiol. 2012 Sep; 78(9):1026-1033. Epub 2012 Jul 6. PMID: 22772860.

6. Delaney, L.J., Van Haren, F. \& Lopez, V (2015). Sleeping on a problem: the impact of sleep disturbance on intensive care patients - a clinical review. Ann. Intensive Care 5, 3 (2015).

7. Devlin JW, Bhat S, Roberts RJ, Skrobik Y (2011). Current perceptions and practices surrounding the recognition and treatment of delirium in the intensive care unit: a survey of 250 critical care pharmacists from eight states. Ann Pharmacother. 2011 Oct; 45(10):1217-1229. doi: 10.1345/aph.1Q332. Epub 2011 Sep 20.

8. Frontera JA (2011). Delirium and sedation in the ICU Neurocrit Care. 2011 Jun; 14(3):463-474. doi: 10.1007/s12028011-9520-0. PMID: 21360232.

9. Galanakis C, Mayo NE, Gagnon B (2011). Assessing the role of hydration in delirium at the end of life. Curr Opin Support Palliat Care. 2011 Jun; 5(2):169-173. doi: 10.1097/SPC.0b013e3283462fdc. PMID: 21532351.

10. Girard TD, Jackson JC, Pandharipande PP, Pun BT, Thompson JL, Shintani AK, Gordon SM, Canonico AE, Dittus RS, Bernard GR, Ely EW (2010). Delirium as a predictor of longterm cognitive impairment in survivors of critical illness. Crit Care Med. 2010 Jul; 38(7):1513-1520. doi: 10.1097/CCM.0b013e3181e47be1. PMID: 20473145; PMCID: PMC3638813.

11. Hipp DM, Ely EW (2012). Pharmacological and nonpharmacological management of delirium in critically ill patients. Neurotherapeutics. 2012 Jan; 9(1):158-175. doi: 10.1007/s13311-011-0102-9. PMID：22270810; PMCID: PMC3271151.

12. Hopkins RO, Suchyta MR, Farrer TJ, Needham D (2012). Improving post-intensive care unit neuropsychiatric outcomes: understanding cognitive effects of physical activity. Am J Respir Crit Care Med. 2012 Dec 15; 186(12):1220-1228. doi: 10.1164/rccm.201206-1022CP. Epub 2012 Oct 11. PMID: 23065013

13. Inouye SK, Westendorp RG, Saczynski JS (2014). Delirium in elderly people. Lancet. 2014; 383(9920):911-922. doi:10.1016/S0140-6736(13)60688-1.

14. Jones SF, Pisani MA (2012). ICU delirium: an update. Curr Opin Crit Care. 2012 Apr; 18(2):146-151. doi: 10.1097/MCC.0b013e32835132b9. PMID: 22322260.Lorenzo, Aldecoa, \& Rico, 2013).

15. Kalabalik J, Brunetti L, El-Srougy R (2013). Intensive care unit delirium: a review of the literature. J Pharm Pract. 2014 Apr; 
27(2):195-207. doi: 10.1177/0897190013513804. Epub 2013 Dec 10. PMID: 24326408.

16. Kamdar BB, Needham DM, Collop NA (2012). Sleep deprivation in critical illness: its role in physical and psychological recovery. J Intensive Care Med. 2012; 27(2):97111. doi:10.1177/0885066610394322

17. Kazmierski J, Banys A, Latek J, Bourke J, Jaszewski R (2013). Cortisol levels and neuropsychiatric diagnosis as markers of postoperative delirium: a prospective cohort study. Crit Care. 2013; 17(2):R38. Published 2013 Mar 1. doi:10.1186/cc12548.

18. Khalil, NS., Moustafa MF \& , El-Bouraei ZM and Shehab ElDeen AHA (2019). Effects of Earplugs and Eye Masks on the Onset of Delirium in a Neuro-Critical Care Unit, Egypt. Nurs Health Care Int J 2019, 3(4): 000196.

19. Khalil, N. S., Moustafa, M. F. \& El-Bouraei, Z. H. (2019). Effects of non-therapeutic measures on sleep quality among critically ill patients, Egypt. International Academic Journal of Health, Medicine and Nursing, 1(2), 175-188.

20. Kim DW, Kim HK, Bae EK, Park SH, Kim KK (2015). Clinical predictors for delirium tremens in patients with alcohol withdrawal seizures. Am J Emerg Med. 2015 May; 33(5):701704. doi: 10.1016/j.ajem.2015.02.030. Epub 2015 Feb 23. PMID: 25745798.

21. Kim H, Chung S, Joo Y H, Lee J S. (2016). The major risk factors for delirium in a clinical setting, Neuropsychiatric Disease and Treatment. (12), 1787-1793.

22. Linda D. Urden, DNSc, RN, CNS, NE-BC, FAAN, Kathleen M. Stacy, (2018) .Critical Care Nursing: Diagnosis and Management, 8th Edition.

23. Lorenzo M. Aldecoa C. Rico J (2013). Delirium in the critically ill patient.Trends Anaesth Crit Care. 2013; 3: 257-264

24. McKinley S, Fien M, Elliott R, Elliott D (2013). Sleep and psychological health during early recovery from critical illness: an observational study. J Psychosom Res. 2013 Dec; 75(6):539545. doi: 10.1016/j.jpsychores.2013.09.007. Epub 2013 Oct 5. PMID: 24290043

25. Needham DM, Korupolu R, Zanni JM, Pradhan P, Colantuoni E, Palmer JB, Brower RG, Fan E (2010). Early physical medicine and rehabilitation for patients with acute respiratory failure: a quality improvement project. Arch Phys Med Rehabil. 2010 Apr; 91(4):536-542. doi: 10.1016/j.apmr.2010.01.002. PMID: 20382284.
26. Pun BT, Boehm L (2011). Delirium in the intensive care unit: assessment and management. AACN Adv Crit Care. 2011 JulSep; 22(3):225-237. doi: 10.1097/NCI.0b013e318220c173. PMID: 21808158

27. Reade MC, Finfer S (2014). Sedation and delirium in the intensive care unit. N Engl J Med. 2014 Jan 30; 370(5):444454. doi: 10.1056/NEJMra1208705. PMID: 24476433.

28. Salluh JI, Wang H, Schneider EB, Nagaraja N, Yenokyan G, Damluji A, Serafim RB, Stevens RD (2015). Outcome of delirium in critically ill patients: systematic review and metaanalysis. BMJ. 2015 Jun 3; 350:h2538. doi: 10.1136/bmj.h2538. PMID: 26041151; PMCID: PMC4454920.

29. Schreiber MP, Colantuoni E, Bienvenu OJ, Neufeld KJ, Chen KF, Shanholtz C, Mendez-Tellez PA, Needham DM (2014). Corticosteroids and transition to delirium in patients with acute lung injury. Crit Care Med. 2014 Jun; 42(6):1480-1486. doi: 10.1097/CCM.0000000000000247. PMID: 24589640; PMCID: PMC4028387.

30. Schweickert WD, Pohlman MC, Pohlman AS, Nigos C, Pawlik AJ, Esbrook CL, Spears L, Miller M, Franczyk M, Deprizio D, Schmidt GA, Bowman A, Barr R, McCallister KE, Hall JB, Kress JP (2009). Early physical and occupational therapy in mechanically ventilated, critically ill patients: a randomised controlled trial. Lancet. 2009 May 30; 373(9678):1874-1882. doi: 10.1016/S0140-6736(09)60658-9. Epub 2009 May 14. PMID: 19446324

31. Weinhouse C, Anderson OS, Bergin IL, Vandenbergh DJ, Gyekis JP, Dingman MA, Yang J, Dolinoy DC (2014). Dosedependent incidence of hepatic tumors in adult mice following perinatal exposure to bisphenol A. Environ Health Perspect. 2014 May; 122(5):485-491. doi: 10.1289/ehp.1307449. Epub 2014 Feb 3. PMID: 24487385; PMCID: PMC4014767.

32. Zaal IJ, Devlin JW, Peelen LM, Slooter AJ (2015). A systematic review of risk factors for delirium in the ICU. Crit Care Med. 2015 Jan; 43(1):40-47. doi:10.1097/CCM.0000000000000625. PMID: 25251759.

33. Zhang, W. Y., Wu, W. L., Gu, J. J., Sun, Y., Ye, X. F., Qiu, W. J., ... Ye, W. Q. (2015). Risk factors for postoperative delirium in patients after coronary artery bypass grafting: A prospective cohort study. Journal of Critical Care, 30, 606-612. https://doi.org/10.1016/j.jcrc.2015.02. 003. 